\title{
Rayleigh Scattering Density Measurements from Ultrafast Lasers in High-Pressure, Cryogenic Wind Tunnels
}

\author{
Ross A. Burns ${ }^{1}$ and Paul M. Danehy ${ }^{2}$ \\ NASA Langley Research Center, Hampton, VA, USA, 23681
}

\begin{abstract}
The Rayleigh scattering signal from femtosecond laser pulses is examined for its utility at making instantaneous density measurements in the NASA Langley 0.3-m Transonic Cryogenic Tunnel. An electron-multiplying CCD camera is used to visualize Rayleigh scattering signal taken concurrently with velocity measurements utilizing the femtosecond laser tagging velocimetry technique (FLEET). The results indicate a strong potential for making instantaneous measurements. Viable single-shot images are obtained over the full operational envelope of the facility, and shot-to-shot variations are found to be on average 6 percent (at 95 percent confidence level) and tend to decrease as the facility density is increased. The Rayleigh scattering signals observed before the optical focus exhibit a characteristically linear dependence on the mass-density of the gas, while signals after the focus exhibit a nonlinear (sublinear) density dependence, indicative of stronger absorption at higher densities. The measured Rayleigh scattering signals compare favorably to theoretical assessments made at the tunnel operating conditions.
\end{abstract}

\section{Introduction}

$\mathrm{O}$ PTICAL measurements in high-pressure, cryogenic wind tunnels have proven difficult since their inception. The rugged construction, limited optical access, oxygen-free operating conditions, and potential for condensation/frost and large density variations over long path lengths that are characteristic of such facilities are often prohibitive to the application of most conventional optical techniques. Over time, special cryogenic variants and modifications to familiar optical diagnostics have enabled modest, though limited successful applications. Temperature sensitive paints have been used prominently for the study of laminar-to-turbulent transition in transonic cryogenic tunnels (TCTs) ${ }^{1,2}$ Reliable cryogenic versions of pressure sensitive paints to measure surface pressure distributions are still in development, ${ }^{3}$ though modern, lifetime-based variants have shown significantly improved sensitivity than older formulations. ${ }^{4}$ Velocity measurements have proven challenging in high-pressure, cryogenic wind tunnels due to the particle seeding requirements of many common techniques such as particle image velocimetry (PIV) and laser Doppler velocimetry (LDV), which have the potential to cause significant contamination of flow circuits. Successful applications of both PIV $^{5}$ and Doppler global velocimetry ${ }^{6}$ have been completed in the European cryogenic wind tunnels. At the NASA TCT facilities, notably the National Transonic Facility (NTF) and the 0.3-m Transonic Cryogenic Tunnel at NASA Langley Research Center, such particle-based methods are currently prohibited due to the potential for facility contamination. Small-scale studies using LDV $^{7}$ and laser transit anemometry ${ }^{8}$ were completed in the 0.3-m TCT facility, though the tests were not conducted over the full operational envelope of the facility and lacked repeatability due to the inconsistency of naturally occurring seed particles. A more recent body of work by the authors utilized the femtosecond laser electronic excitation tagging (FLEET) technique, ${ }^{9}$ a seedless velocimetry technique based on the excitation of molecular nitrogen with a fs-laser, to make accurate, precise, and reliable velocity measurements over the full operational range of the facility. ${ }^{10,11,12}$

Of particular interest to the present study are the applications of molecular scattering techniques in TCT facilities for use in density measurements. Studies by Shirinzadeh and Herring utilized both continuous wave (CW) and pulsed Nd:YAG lasers at $532 \mathrm{~nm}$ to detect molecular Rayleigh scattering in the 0.3-m TCT facility. ${ }^{13,14}$ Additional studies by Herring et al. studied the feasibility of performing Rayleigh scattering in the NTF. ${ }^{15}$ These studies were able to detect molecular Rayleigh scattering along a line without significant interference from particles using a CW Nd:YAG laser at $532 \mathrm{~nm}$.

\footnotetext{
${ }^{1}$ Research Engineer, National Institute of Aerospace, AIAA Member

${ }^{2}$ Senior Technologist (ST), Advanced Measurement and Data Systems Branch, AIAA Associate Fellow
} 
In this manuscript, the use of molecular Rayleigh scattering from ultrafast laser pulses will be examined for its utility at making absolute or relative density measurements within high-pressure, cryogenic wind tunnels. The principal application of such a technique would be to make simultaneous, time-correlated measurements of density and velocity using both the scattering and FLEET techniques. Such a combination would allow for the direct measurement of Reynolds stresses (e.g., $\left\langle\rho u_{x}^{\prime} u_{x}^{\prime}\right\rangle$ or $\left\langle\rho u_{x}^{\prime} u_{y}^{\prime}\right\rangle$ ) or joint, single-point statistics more generally in either wind tunnel freestreams or turbulent regions of flows. However, there are many challenges to overcome in trying to implement such a measurement. The fundamental wavelength at which most fs-lasers operate, $800 \mathrm{~nm}$, is far from optimal for Rayleigh scattering measurements. The differential scattering cross-section is only a small fraction of those at the visible and ultraviolet wavelengths at which Rayleigh scattering is typically performed (approximately 20 percent compared to $532 \mathrm{~nm}$ and only 0.3 percent compared to $193 \mathrm{~nm}$ ). Moreover, only a small amount of energy $(\sim 1 \mathrm{~mJ})$ can be used with each pulse so that the perturbation of the flow due to the FLEET effect is minimized. A previous attempt at making Rayleigh scattering measurements from fs-laser pulses by the authors was hindered by low signal intensity for these reasons, making instantaneous measurements impossible except at the highest densities encountered. ${ }^{11}$ A different camera technology was utilized in these studies, which offered a significant improvement in both the quantum efficiency of the sensor and the gain applied to the signal. The data resulting from these studies is herein examined for its use in single-shot density measurements. This manuscript is structured as follows. After this introduction, the experimental methodologies are discussed including a discussion of the test facility, laser system, and data acquisition systems. The analytical methods utilized in data reduction are then discussed. The results of the study are then presented, followed by the conclusions. An appendix details calculations used in theoretically estimating the Rayleigh scattering intensity.

\section{Experimental Methodology}

\section{A. Test Facility}

All experiments were conducted in the NASA Langley 0.3-m Transonic Cryogenic Tunnel (0.3-m TCT); a closed-loop, fan-driven wind tunnel capable of operating a Mach numbers ranging from 0.2 to 0.9. While able to operate on a number of test gases, all tests were conducted in nitrogen $\left(\mathrm{N}_{2}\right)$. Operating total (stagnation) pressures ranged from 100 to $500 \mathrm{kPa}$, and the total temperature was varied from $100 \mathrm{~K}$ through $280 \mathrm{~K}$. The facility is constructed with a double-shelled design; the central test section (or test cell) is surrounded by a pressurized plenum of nominally quiescent gas. This construction allowed the presence of either adjustable or slotted walls in the test section. The test section has cross-section dimensions of approximately $0.33 \mathrm{~m} \times 0.33 \mathrm{~m}$. The test section was set to have slightly diverging walls to account for boundary layer growth over most conditions. Optical access to the facility was afforded by two fused silica windows penetrating the plenum and test section. A large, circular window penetrated the outer plenum wall, while a ' $\mathrm{D}$ '-shaped hexagonal window (henceforth referred to as the D-window) was situated in the wall of the inner test section to allow optical access to the inner test cell.

\section{B. Laser System}

The Rayleigh scattering measurements utilized a pulsed, regeneratively-amplified Ti:sapphire laser (SpectraPhysics Solstice) with a center wavelength of $800 \mathrm{~nm}$, bandwidth of $20 \mathrm{~nm}$ FWHM, a pulse duration of $70 \mathrm{fs}$, and a repetition rate of $1 \mathrm{kHz}$. Data sets were collected with pulse energies around $1 \mathrm{~mJ}$ measured at the laser source, although between 30 to 50 percent of this energy was attenuated through the beam path. The laser system was situated on a platform roughly $3 \mathrm{~m}$ above the test section; the beam was brought down to the level of the facility with a pair of periscopic mirrors. To allow for adjustment during facility contraction (a common occurrence when operating at cryogenic conditions), the beam was first routed through a pair of horizontal periscopes that could be translated to keep the beam in alignment. The beam was then transmitted into the plenum of the facility, elevated to the height of the D-window, and passed through a $f=+250 \mathrm{~mm}$ spherical lens before entering the test section. Note that this focusing lens was used to generate the FLEET effect for a separate study; ${ }^{12}$ the Rayleigh scattering measurements were subordinate to the associated velocity measurements during these tests. The laser beam path at the facility level is detailed in Fig. 1. 


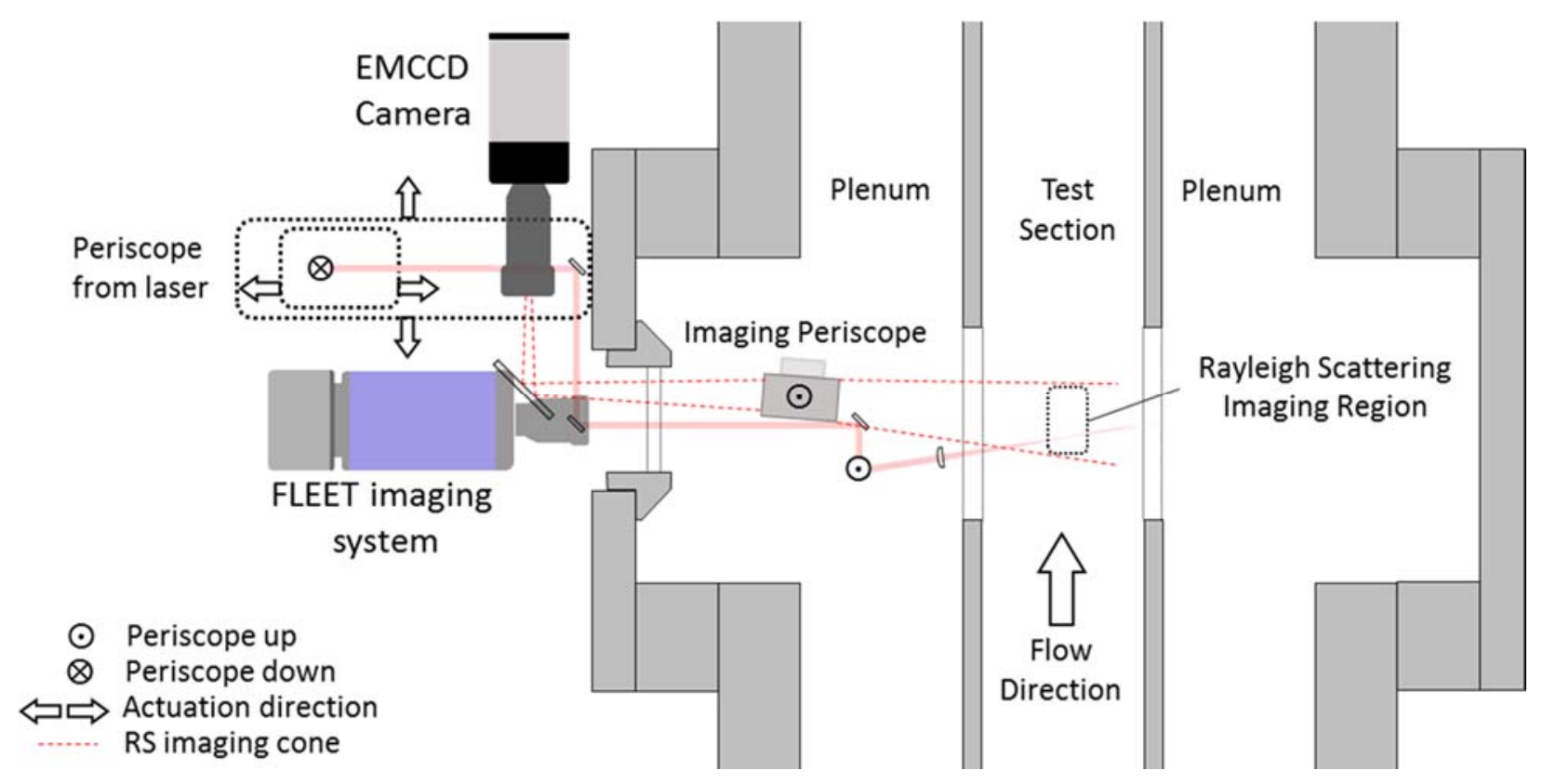

Figure 1. Top view of the laser and optical systems in and around the 0.3-m TCT facility.

\section{Data Acquisition Systems}

The Rayleigh scattering signal was recorded with an electron-multiplying charge-coupled device (EMCCD) camera (Princeton Instruments PhotonMAX 512) equipped with a $185 \mathrm{~mm}, \mathrm{f} / 2.8$ objective lens with the aperture set to $f / 5.6$ to keep the image in focus. This sensor provided low-noise imaging while allowing the application of significant avalanche gain and high quantum efficiency (70-80 percent) at the desired wavelength. A bandpass filter (center wavelength: $800 \mathrm{~nm}$, bandwidth: $25 \mathrm{~nm}$ ) was placed in front of the objective lens to isolate the light from the femtosecond laser pulse. The camera used a $10 \mu$ s exposure to capture the scattering from the laser pulse; the laser pulse was positioned toward the end of the exposure to reduce the amount of signal from the FLEET effect that might have been captured. The image acquisition rate was approximately $30 \mathrm{~Hz}$. The imaging paraphernalia was part of a larger experimental setup used to perform concurrent measurements with FLEET velocimetry, and consequently the Rayleigh scattering imaging path was convoluted, requiring imaging at an oblique angle. Figure 1 shows this Rayleigh scattering imaging path as it was situated in the broader experiment. The camera was positioned anti-parallel to the principal flow direction. The imaging line-of-sight (LOS) was directed through, first, a horizontal mirror to turn the LOS perpendicular to the flow, and second, a vertical periscope that shifted the LOS to the height of the internal D-window while simultaneously rotating it between 5 and 10 degrees. This convolution was necessary to avoid both the laser beam and associated optics as well as the imaging LOS from the other camera system being used for the velocimetry.

In addition to the imaging system, the 0.3-m TCT facility had an extensive data acquisition system (DAS), consisting of an array of wall pressure taps, thermocouples, pitot probes, and pressure transducers to measure relevant properties of the flow. These probes were situated throughout the test section walls and within the plenum. Data from this equipment was read into facility computer systems for processing. In post-processing, additional parameters such as the velocity, density, and Reynolds number were inferred from the pressure and temperature measurements using an equation of state (Beattie-Bridgeman equation ${ }^{16}$ ) and the thermodynamic properties of nitrogen. ${ }^{17} \mathrm{~A}$ facility data point was collected during each Rayleigh scattering data acquisition run to measure the corresponding facility conditions. 


\section{Analytical Methods}

\section{A. Image processing}

A sample raw Rayleigh scattering image from these experiments is shown in Fig. 2. In addition to the Rayleigh scattering signal, a gradient background is also present in all images. To isolate the signal, a numerical background subtraction was performed on the images. The images were initially rotated to orient the scattering signal vertically. A second-order polynomial was then fit to the area surrounding the scattering signal (specifically excluding the signal). This polynomial took the form:

$$
B G_{R S}(y, z)=A_{1}\left(A_{2} z^{2}+A_{3} z+A_{4}\right)\left(A_{5} y^{2}+A_{6} y+A_{7}\right)+A_{8}
$$

Each of the $A_{n}$ terms is fit parameters for the polynomial function. After fitting this polynomial to the image with a least-squares routine, it was subtracted from the rotated image. This procedure was necessary because the background originated from the same laser pulse as the Rayleigh scattering (thus possessing the same wavelength and occurring simultaneously), making it impossible to remove through temporal gating, spectral filtering, or conventional background subtraction. The quality of the background subtraction was then assessed by verifying the average background was at or near zero at various points in the image.

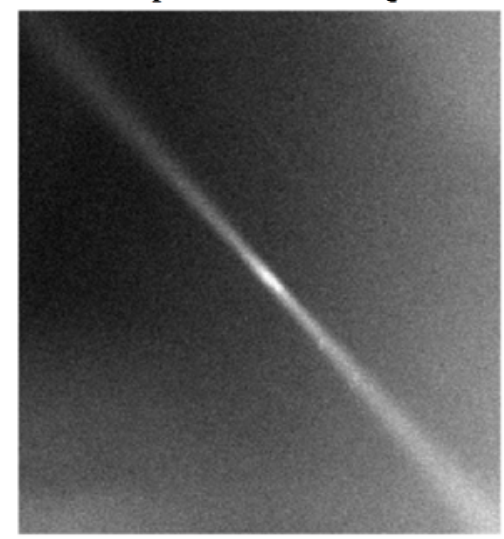

Figure 2. Sample raw Rayleigh scattering image. Conditions are $\mathrm{P}=445 \mathrm{kPa}, \mathrm{T}=271 \mathrm{~K}$, and $\mathrm{M}=0.4$.

\section{B. Signal data reduction}

After background subtraction, the Rayleigh scattering signal was assessed by summing the signal across the width of the laser beam (horizontal with respect to the images). A region $17 \mathrm{px}(1.3 \mathrm{~mm}$ ) wide was used in this summation. While the Rayleigh scattering intensity varies throughout the laser profile and with span-wise distance, the summation across the beam is constant (since the laser fluence is constant). A sample instantaneous profile constructed in this manner is shown in Fig. 3. There is a high-intensity peak at the center of each profile. This signal corresponds to the residual FLEET signal that passed through the bandpass filter. As a result, only the signal on either side of the central intensity peak was considered in the analysis. The included regions are highlighted in red in Fig. 3. An additional step is required for some images, where the presence of larger scatterers (dust, ice crystals, or $\mathrm{LN}_{2}$ droplets, for instance) causes a large local increase in signal intensity. To avoid contamination of the signal from such sources, a signal filter was used to remove such occurrences. This filter functioned by calculating the mean and standard deviation of the signal across all images for a given data run. Any data beyond 2.5 standard deviations of the mean were removed from the signal profiles, and the mean and standard deviation were recalculated. The procedure was then repeated, and the resulting signal used in analysis. Roughly 10 percent of runs contained large numbers of scatterers, but less than 2 percent of all data were removed through this procedure, since it only removes signal locally. After the signal profiles were filtered, the span-wise mean of the signal was then compared to the gas density inferred from facility DAS measurements. 


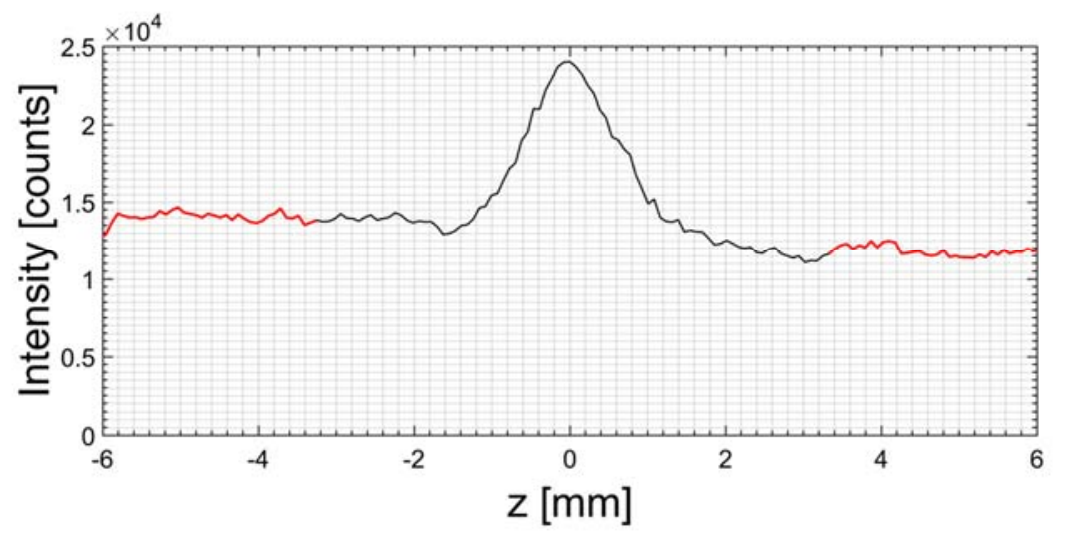

Figure 3. Span-wise Rayleigh scattering intensity profile after processing. Red regions are those used in evaluating the data.

\section{Results and Discussion}

One concern surrounding these experiments was that the Rayleigh scattering signal would be too weak to make single-shot measurements, which are necessary for making density measurements instantaneously correlated with velocity. The previous attempt at using this Rayleigh scattering as an instantaneous measurement showed low signal counts, even when 4-shot accumulations were used. ${ }^{11}$ However, the EMCCD sensor utilized in the current study had a quantum efficiency roughly 2.5 times higher than that of the sCMOS sensor used previously with comparable noise levels. This sensor also allowed for significant on-chip gain, with a factor of roughly 160 applied during these tests as inferred from the product specifications.

Figure 4 shows instantaneous and mean Rayleigh scattering images taken at different pressures. Note that these images have been processed according to the same procedure described previously in Section III. These images show the convergence and divergence of the fs-laser beam near the focus; the beam enters the images from the top, and the bright spot within the focal region is both scattering from the laser as well as a component of the broadband emission from either photo-ionization of the gas or short-lived FLEET signal nearly resonant with the laser.

The instantaneous images shown in the top panes of Fig. 4 indicate that there is usable signal within the entirety of the imaging field of view, even at the lowest tested pressures (roughly the lowest operating pressure in the facility, which is slightly above an atmosphere). The observed signal became progressively higher as the pressure (and consequently the density) was increased to the facility maximum. The shot-to-shot variations of the measured scattering intensity between the instantaneous images were approximately 6 percent (based on $2 \sigma$, or $95 \%$ confidence) on average and tended to decrease as the pressure was increased, likely due to the higher signals observed at these conditions. The higher signal leads to a greater overall signal-to-noise ratio in the images since the read noise is roughly constant, and the shot noise scales as the square root of the signal intensity. These results are a dramatic improvement over the previous attempts at using Rayleigh scattering in this facility with the fs-laser, which required averaging to make the signals usable over most of the operational envelope of the facility. The shot-to-shot variations observed may also be lessened (improved) by applying a correction for the laser intensity variations, which was not done in these studies. Rather, we assumed that the laser pulse intensity was constant shot to shot; although pulse energy variations between 1 and 2 percent are common. Note from the average images in the bottom panes of Fig. 4 that the signal-to-noise ratio does improve significantly by ensemble-averaging, indicating that the mean images could be used if the signals were found to decrease to unusable levels in the instantaneous images (if simultaneous measurements were not the intent).

Figure 5a shows the measured Rayleigh scattering signal intensity taken before the focus of the beam as a function of the facility operating density, which was inferred from the facility DAS measurements. The signal that is plotted in Fig. 5 was constructed as described in Section III.B. The color coding indicates the static temperature, and the error bars $(2 \sigma)$ indicate the shot-to-shot variations in the instantaneous images at the tested conditions. Overall the observed trend is linear in form, as is expected with molecular Rayleigh scattering. While unlikely, it is possible that clustering or gaseous condensation might have resulted in scattering from very small particles (still in the Rayleigh regime). To assess whether this occurred, theoretical calculations of the expected Rayleigh scattering 


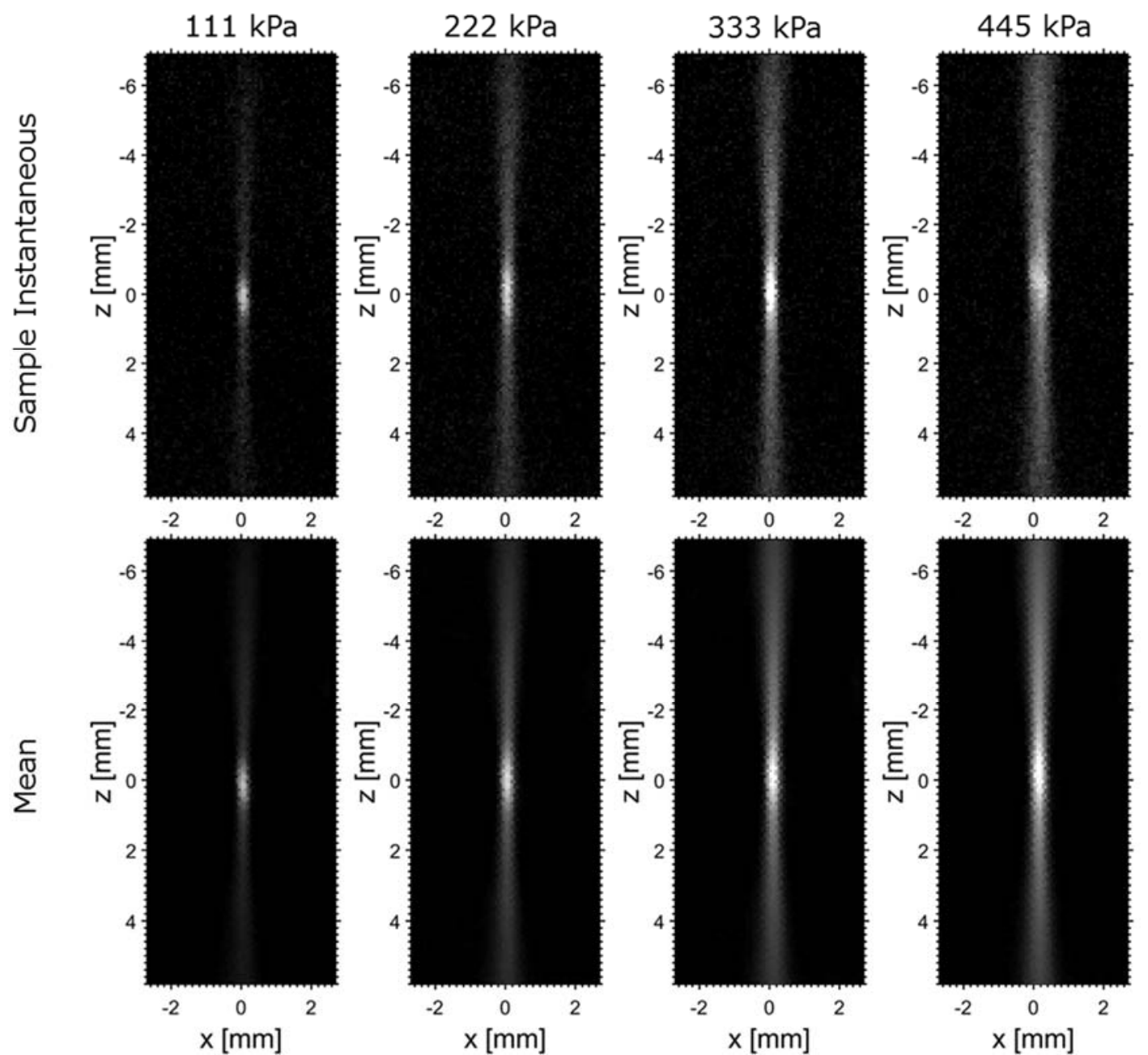

Figure 4. Sample instantaneous and mean Rayleigh scattering images taken at four different pressures. Mach 0.4 and $\mathrm{T}=228 \mathrm{~K}$ for all conditions.

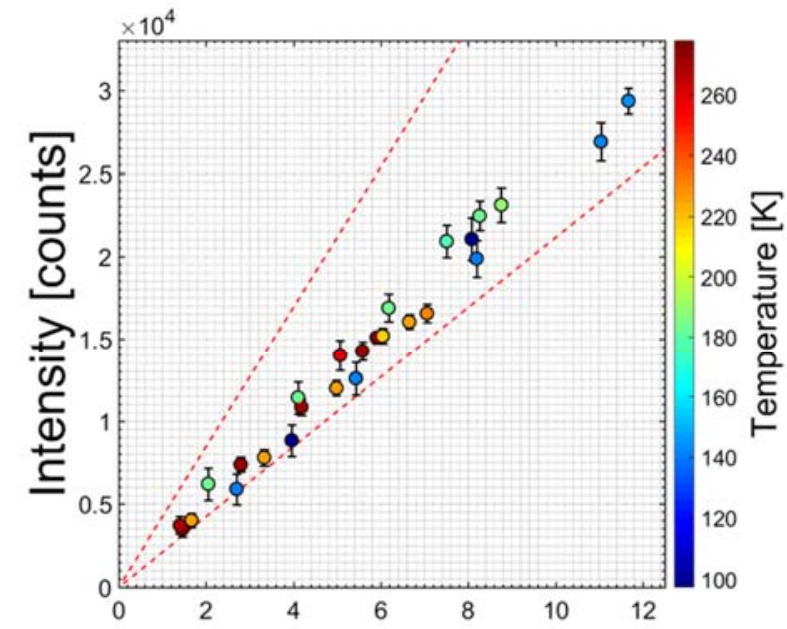

a)

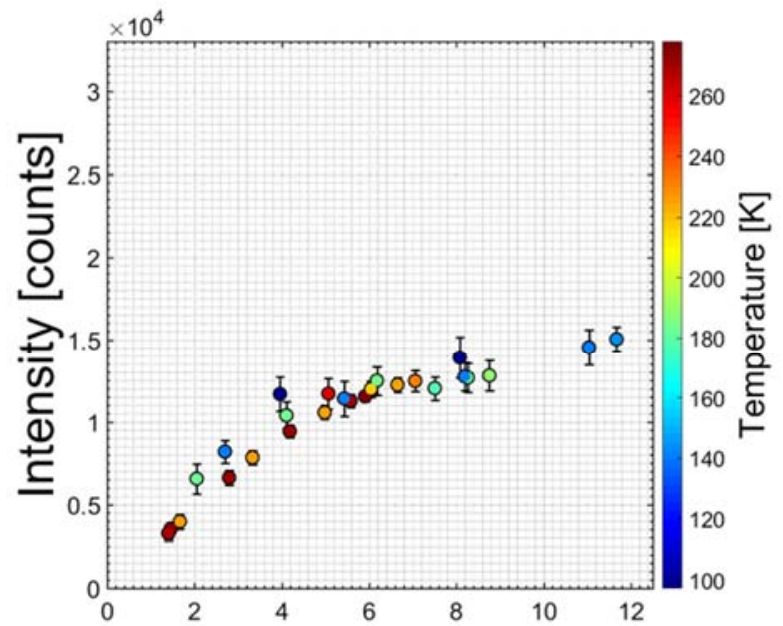

b)

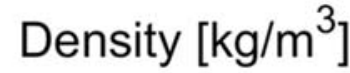

Figure 5. Rayleigh scattering signal dependence on operating density. a) Signal before optical focus and b) signal after optical focus. Dashed lines in a) represent the upper and lower limits of the predicted Rayleigh scattering intensity. 
signal intensity were performed based on the experimental parameters. While the details of these calculations are discussed in Appendix 1, the results of the calculations are shown in Fig. 5a, with the upper and lower bounds of the expected signal denoted by dashed red lines. As is demonstrated by this comparison, the measured Rayleigh scattering signal falls clearly within the uncertainty bounds of the theoretical estimates, though on the low end of the estimated range. It should be stated for clarity that the uncertainty in the Rayleigh scattering signal calculations stems predominantly from the degree of attenuation of the laser beam and the uncertainty in the collection solid angle of the lens/mirror system used to acquire the data. Nonetheless, this assessment indicates that the Rayleigh scattering signal is within theoretical estimates, indicating that it is molecular in origin.

A different behavior is observed in the signal dependence if the scattering signal after the optical focus is used in these same analyses. Rather than exhibiting a linear dependence on the density as the region before the focus had, the signal is found to grow less rapidly with density; this trend is shown in Fig. 5b. At the lowest densities examined in these tests, the Rayleigh scattering signals after the focus roughly achieve parity with those before. However, at the highest densities, only half of the signal intensity is observed. The primary factor that results in this effect is absorption; passing through the focus and producing the FLEET effect, inducing the photo-ionization of nitrogen, and continuum generation (as well as any other non-linear processes that occur) collectively result in part of the incident laser energy being absorbed and attenuated. These results indicate that the ratio of the two signals before and after the focus, which is a measurement of the overall transmission, could potentially be used as an absorption measurement. The decrease in transmission through the focus as the density is increased may furthermore imply greater absorptivity (or absorption cross-section) and possibly greater localized heating of the flow by the fs-laser pulse as a mechanism and outcome. Further studies would be required to ascertain the specific relationship between the observed change in transmission and the absorptivity of the gas through the focal region.

One principal drawback to the present Rayleigh scattering measurements, despite the acceptable signal levels, is the non-locality required to avoid the bright signal near the optical focus of the fs-laser beam. Because of this, the 2$3 \mathrm{~mm}$ long region that contains this signal cannot be considered in the calculations, and thus the density measurements are made in a region explicitly excluding the area where the velocity measurement is being made. If it was desired to make simultaneous velocity and density measurements, the effective spatial resolution of the technique would have to be classified as much broader than that of the velocity or density measurements alone to accommodate this effect. One possible strategy to improve a simultaneous measurement would be to use a narrower spectral filter, which would reduce the overall contribution of the FLEET signal to the Rayleigh scattering signal near the focus while still transmitting most of the laser light. Since the degree of the contribution from the FLEET signal is not currently known, it is unclear how effective this strategy would be in practice. Another strategy, though more complicated, could be to use a different harmonic of the fs-laser beam; the (usual) fundamental wavelength of $800 \mathrm{~nm}$ is close to several of the FLEET emission lines, invariably leading to contamination of the FLEET signal. If, alternatively, a much shorter wavelength were used for excitation (for instance, $202 \mathrm{~nm}$ used for the STARFLEET technique ${ }^{18}$ ), much of this secondary emission could be avoided and the scattering signal could potentially be isolated from the FLEET signal, allowing a coincident measurement to be made. Although the differential Rayleigh scattering cross-section is significantly larger at this wavelength, the quantum efficiency of many image sensors decreases precipitously below $300 \mathrm{~nm}$, so the increase in signal intensity would likely be mitigated to an extent. Also, STARFLEET uses much lower energy levels than conventional FLEET (roughly a factor of 30), further decreasing the available signal. ${ }^{18}$ If an improved scattering measurement were desired and performing it concurrently with a velocity measurement was not necessary, the second harmonic of the beam could be used to increase the signal substantially. Additionally, intentionally not focusing the beam would altogether avoid the formation of the FLEET signal and allow the density to be properly imaged along a line with minimal adjustment to the current experimental setup.

\section{Conclusions}

A series of experiments was conducted to evaluate Rayleigh scattering from low-energy, fs-laser pulses for gas density measurements. These experiments were conducted concurrently with FLEET velocity measurements, which utilized the same laser pulse. A linear signal dependence was observed prior to the fs-laser beam focus, consistent with molecular scattering. The observed trend compared favorably with theoretical estimates of the molecular scattering intensity. After the optical focus, a sublinear dependence on the density was observed in the signal intensity, likely indicative of the degree of absorption occurring within the focal region. These results indicate that Rayleigh scattering from low-energy fs-laser pulses has the potential to serve as an instantaneous indicator of flow 
density that is correlated with simultaneous FLEET velocity measurements, yielding time-correlated, multiparameter measurements. Instantaneous signal levels were sufficiently high over the full operational envelope of the NASA $0.3-\mathrm{m}$ TCT facility to enable instantaneous ( 100 fs duration) single-laser-pulse measurements.

\section{Appendix I. Theoretical Rayleigh Scattering Intensity Estimations}

In order to estimate the expected Rayleigh scattering signal intensity to confirm the molecular origin of the observed signals, a theoretical model of the experiment was constructed. The simulations started by modeling the equipment used in the experiments. The fs-laser pulse had a center wavelength of $800 \mathrm{~nm}$ and a bandwidth of $20 \mathrm{~nm}$ (full width at half-maximum). Figure A1.a shows the initial laser spectrum used in these simulations. This broad laser line complicates the calculation of the Rayleigh scattering intensity because the Rayleigh scattering crosssection, index of refraction, transmission of the collection bandpass filter, and quantum efficiency of the camera sensor all have non-trivial wavelength dependences. The collection bandpass filter had a center wavelength of 800 $\mathrm{nm}$ and a pass-band $25 \mathrm{~nm}$ wide; the transmission of this filter is shown in Fig. A1.b. ${ }^{19}$ The EMCCD sensor quantum efficiency varied from roughly 90 percent at $700 \mathrm{~nm}$ to 38 percent at $900 \mathrm{~nm}$. Figure A1.c shows the wavelength dependence of the sensor quantum efficiency. ${ }^{20}$

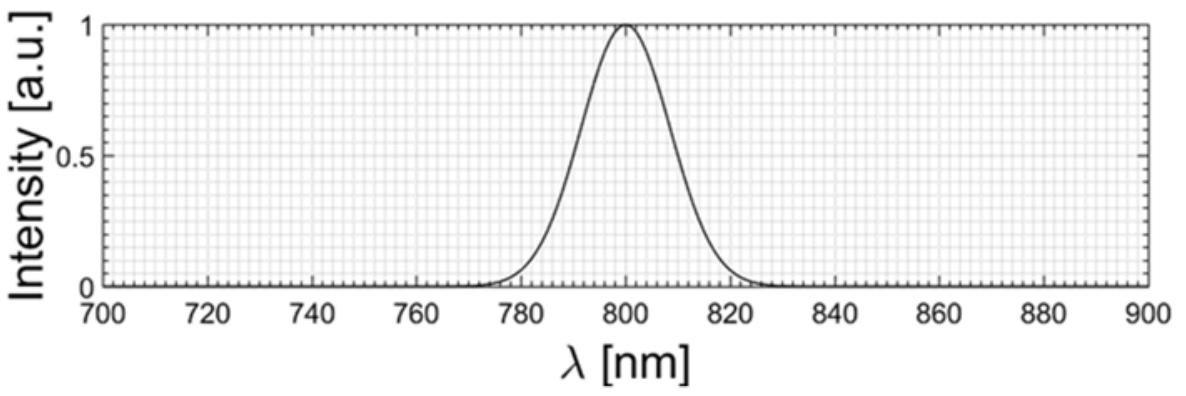

a)

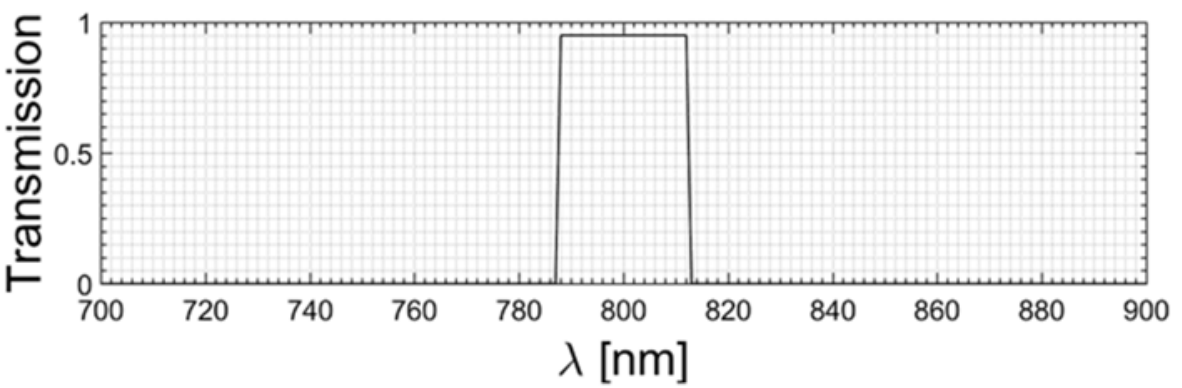

b)

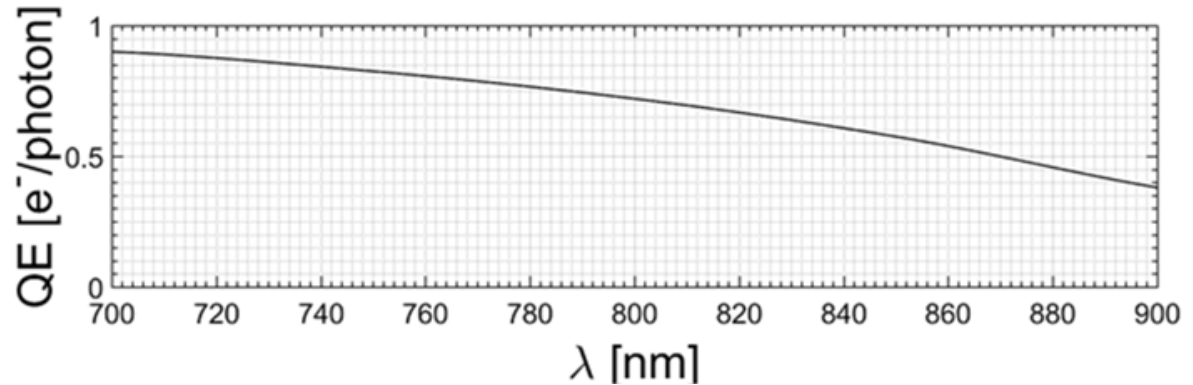

Figure A1. Rayleigh scattering simulation parameters. a) Initial laser spectrum, b) bandpass filter transmission curve, and c) EMCCD sensor quantum efficiency.

Calculating the Rayleigh scattering intensity requires the evaluation of the observed Rayleigh scattering crosssection. The total Rayleigh scattering cross-section is given by Eq. A.1, while the differential cross-section is given by Eq. A.2: ${ }^{21,22,23}$

$$
\sigma_{R S}=\frac{24 \pi^{3}}{\lambda^{4} N^{2}}\left(\frac{n^{2}-1}{n^{2}+2}\right)^{2} F_{k}
$$




$$
\frac{\partial \sigma_{R S}}{\partial \Omega}=\frac{9 \pi^{2}}{\lambda^{4} N^{2}}\left(\frac{n^{2}-1}{n^{2}+2}\right)^{2} F_{k} \sin ^{2} \phi
$$

In Eqs. A. 1 and A.2, $N$ is the number density of the gas, $n$ is the real index of refraction of the gas, $F_{k}$ is the effective King correction factor, which is associated with the depolarization caused by the non-spherical charge distribution of molecular nitrogen. The angle, $\phi$, is the angle between the direction of observation and the direction of the induced dipole oscillation in individual molecules. The differential cross-section differes from the total crosssection in that it has not been spatially integrated over a particular collection volume. Each collection system subtends a slightly different portion of the entire volume in which scattering can be observed. Consequently, the observed cross-section (the intergral of the differential cross-section with respect to the collection system) will always be less than or equal to the total cross-section. Further, the index of refraction (Eq. A.3, Ref. 24) and King correction factor (Eq. A.4, Refs. 22,25,26, and 27) for nitrogen can be expressed as:

$$
\begin{gathered}
n=1+\left[6498.2+\frac{307.43305 \times 10^{12}}{14.4 \times 10^{9}-\bar{v}^{2}}\right]\left(\frac{\rho}{\rho_{o}}\right) \times 10^{-8} \\
F_{k}=1.034+3.17 \times 10^{-12} \bar{v}^{2}
\end{gathered}
$$

Equations A.3 and A.4 rely on the wavenumber $\left(\bar{v}\left[\mathrm{~cm}^{-1}\right]\right)$ rather than the wavelength. Additionally, the index of refraction has a density dependence, which is referenced in Eq. A.3 against the density at STP, $\rho_{o}$.

Procedurally, the simulations were initiated by calculating how many photons were present at each wavelength. This was done by first specifying the total initial pulse energy $(1 \mathrm{~mJ})$ and multiplying the spectral intensity of the laser pulse by the photon energy at each wavelength. The product of the spectral intensity and the photon energy was then summed over all wavelengths, and the total pulse energy was divided by this quantity to find a scaling factor. The number of photons at each wavelength was calculated as the initial spectral intensity multiplied by this scaling factor. The simulations then continued by specifying a gas density and calculating the differential crosssection for each wavelength considered. The observed scattering cross-section, $\sigma_{R S, o b s}$, was then calculated by integrating Eq. A.2. over the observation solid angle. The ratio between the observed cross-section and total crosssection was roughly 8 percent, with a large (roughly a factor of 2) uncertainty due to the overall geometric uncertainty in the optical setup. The number of observed photons at the camera sensor was then calculated as:

$$
N_{\gamma}(\lambda)=N_{\gamma, o}(\lambda) T_{o p t} T_{\text {filt }}(\lambda) \Delta z N \sigma_{R S, o b s}
$$

$N_{\gamma}$ and $N_{\gamma, o}$ in Eq. A.5 are the current and initial number of photons at each wavelength, $T_{o p t}$ is the transmission of the optical system, which includes losses associated with the broadband mirrors and losses at each optical interface (8 surfaces total in the experimental setup) estimated lie between 42 and 72 percent, $T_{\text {filt }}$ is the transmission of the collection bandpass filter (as a function of wavelength), and $\Delta z$ is the width of the flow subtended by a single pixel on the sensor. This formulation sums the photons scattered within thin cross-sections of the conical, converging laser profile. Implicitly assumed in this formulation is that the laser has a uniform spatial intensity profile and further that the observed scattering cross-section does not vary substantially within the laser volume. While the laser does not have a uniform profile in practice, the summation through the beam cross-section averages out to the same number of photons and was an assumption made to simplify the analysis. Finally, the signal was calculated as:

$$
I_{R S}=\frac{G D}{C_{F W}} \int_{\lambda} Q E(\lambda) N_{\gamma}(\lambda) d \lambda
$$

In Eq. A.6, $G$ is the on-chip gain of the sensor (160), $C_{F W}$ is the full-well capacity of each pixel (800 $\left.e^{-}\right), D$ is the maximum number of counts for each pixel (16-bit, 65535), and the integral is the total number of photoelectrons initially freed on the EMCCD before gain. In the actual experiment, the signal was distributed over 17 pixels and was then summed, whereas in the simulations the sum over the beam cross-section was calculated first and was then translated to pixel counts. Ultimately these processes are the same, but the simulations simplify the optical setup 
rather than modeling the scattering in a pixel-wise fashion. These calculations were then repeated for several densities ranging from $0 \mathrm{~kg} / \mathrm{m}^{3}$ to $15 \mathrm{~kg} / \mathrm{m}^{3}$ and for the range of system transmissivities $\left(T_{\text {opt }}\right)$. The upper and lower limits of the estimated signal intensity are presented in Figure. 5a along with the signal encountered in the experiment.

\section{Acknowledgements}

The authors would like to thank all of the expert staff at the NASA Langley $0.3-\mathrm{m}$ TCT including Wes Goodman, Michael Chambers, Karl Maddox, Cliff Obara, Chris Cramer, Reggie Brown, Gary Beachem, and Lynn Bobbitt. These tests would not have been possible without the long hours and hard work everyone contributed. This work was supported by NASA Langley's Internal Research and Development (IRAD) program and NASA's Aeronautics Research Mission Directorate (ARMD) Aeronautics Evaluation and Test Capability (AETC) Project.

\section{References}

${ }^{1}$ Fey, U., Konrath, R., Kirmse, T., Ahlefeldt, T., Kompenhans, J., and Egami, Y., "Advanced Measurement Techniques for High Reynolds Number Testing in Cryogenic Wind Tunnels," 48th AIAA Aerospace Sciences Meeting Including the New Horizons Forum and Aerospace Exposition, Orlando, FL, 2010, pp. 1-8. AIAA-20101301.

${ }^{2}$ Fey, U., Egami, Y., and Engler, R.H., "High Reynolds number transition detection by means of temperature sensitive paint," 44 $4^{\text {th }}$ AIAA Aerospace Sciences Meeting and Exhibit, Reno, NV, 2006, pp. 1-19. AIAA-2006-514.

${ }^{3}$ Watkins, A.N., Leighty, B.D., Lipford, W.E., Oglesby, D.M., Goodman, K.Z., Goad, W.K., Goad, L.R., Massey, EA., "The Development and Implementation of a Cryogenic Pressure Sensitive Paint System in the National Transonic Facility," 47th AIAA Aerospace Sciences Meeting including The New Horizons Forum and Aerospace Exposition,” Orlando, FL, 2009, pp. 1-10, AIAA-2009-421.

4 Yorita, D., Klein, C., Henne, U., Ondrus, V., Beifuss, U., Hensch, A.K., Guntermann, P., and Quest, J., "Application of Lifetime-based Pressure-Sensitive Paint Technique to Cryogenic Wind Tunnel Tests," 54 ${ }^{\text {th }}$ AIAA Aerospace Sciences Meeting, San Diego, CA, 2016.

${ }^{5}$ Quest, J. and Konrath, R. "Accepting a Challenge - The Development of PIV for Application in pressurized cryogenic Wind Tunnels," 41st AIAA Fluid Dynamics Conference and Exhibit, Honolulu, HI, 2011.

${ }^{6}$ Willert, C., Stockhausen, G., Beversdorff, M., Klinner, J., Lempereur, C., Barricau, P., Quest, J., and Jansen, U., "Application of Doppler global velocimetry in cryogenic wind tunnels," Experiments in Fluids, Vol. 39, 2005, pp. 420-430.

${ }^{7}$ Gartrell, L. R., Gooderum, P. B., Hunter, W. W., and Meyers, J. F., "Laser Velocimetry Technique Applied to the Langley 0.3-Meter Transonic Cryogenic Tunnel," NASA TM- 81913, 1981.

${ }^{8}$ Honaker, W. C. and Lawing, P. L., "Measurements in the Flow Field of a Cylinder with a Laser Transit Anemometer and a Drag Rake in the Langley 0.3-m Transonic Cryogenic Tunnel," NASA TM- 86399, 1985.

${ }^{9}$ Michael, J. B., Edwards, M. R., Dogariu, A., and Miles, R. B., "Femtosecond laser electronic excitation tagging for quantitative velocity imaging in air," Applied Optics, Vol. 50, No. 26, 2011, pp. 5158-5162.

${ }^{10}$ Burns, R.A., Danehy, P.M., Halls, B.R., and Jiang, N., "Unseeded Velocimetry in a Transonic, Cryogenic Wind Tunnel Using FLEET,” AIAA Journal, pp. 1-6.

${ }^{11}$ Burns, R.A., Peters, C.J., and Danehy, P.M., "Femtosecond-Laser-Based Measurements of Velocity and Density in the NASA Langley 0.3-m Transonic Cryogenic Tunnel," 32 ${ }^{\text {nd }}$ AIAA Aerodynamic Measurement Technology and Ground Testing Conference, Washington, D.C., 2016, AIAA 2016-3246.

12 Burns, R.A. and Danehy, P.M., "Unseeded Velocity Measurements Around a Transonic Airfoil Using Femtosecond-Laser Tagging," AIAA Journal, in publication.

${ }_{13}$ Shirinzadeh, B., Herring, G. C., and Barros, T. "Demonstration of Imaging Flow Diagnostics Using Rayleigh Scattering in Langley 0.3-Meter Transonic Cryogenic Tunnel," NASA Technical Note 1999-208970, Hampton, VA, 1999.

${ }^{14}$ Herring, G.C. and Shirinzadeh, B., "Flow Visualization of Density in a Cryogenic Wind Tunnel Using Planar Rayleigh and Raman Scattering,” NASA TM-2002-211630, 2002.

${ }^{15}$ Herring, G.C., Lee, J.W., and Goad, W.K., "Feasibility of Rayleigh Scattering Flow Diagnostics in the National Transonic Facility,” NASA TM-2015-218800, 2015. 
${ }^{16}$ Beattie, J.A. and Bridgeman, O.C., “A New Equation of State for Fluids,” Proceedings of the American Academy of Arts and Sciences. Vol. 63, No. 5, 1928, pp. 229-308.

17 Jacobsen, R.T., and Stewart, R.B., "Thermodynamic Properties of Nitrogen Including Liquid and Vapor Phases from 63 K to 2000 K with Pressures to 10,000 Bar,” Journal of Physical Chemistry Reference Data, Vol. 2, No. 4, 1973, pp. 757-922.

18 Jiang, N., Halls, B.R., Stauffer, H.U., Danehy, P.M., Gord, J.M, and Roy, S., "Selective two-photon absorptive resonance femtosecond-laser electronic-excitation tagging velocimetry,” Optics Letters, Vol. 41, No. 10, 2016, pp. 2225-2228.

${ }^{19}$ Coating Curve, Bandpass filter 86672, Edmund Optics Inc.

${ }^{20}$ PhotonMAX 512 product specifications, Princeton Instruments.

21 Miles, R.B., Lempert, W.R., and Forkey, J.N., “Laser Rayleigh Scattering," Measurement Science and Technology, Vol. 12, No. 5, 2001, pp. R33-R51.

${ }^{22}$ Sneep, M. and Ubachs, W., "Direct Measurement of the Rayleigh scattering cross section in various gases," Journal of Quantitative Spectroscopy and Radiative Transfer, Vol. 92, No. 3, 2004, pp. 293-310.

${ }^{23}$ Thalman, R., Zarzana, K.J., Tolbert, M.A., and Volkamer, R., "Rayleigh scattering cross-section measurements of nitrogen, argon, oxygen, and air,” Journal of Radiative Spectroscopy and Radiative Transfer, Vol. 147, 2014, pp. 171-177.

${ }^{24}$ Peck, E.R. and Khanna, B.N., "Dispersion of Nitrogen,” Journal of the Optical Society of America, Vol. 56, No. 8, 1966, pp. 1059-1063.

${ }^{25}$ Alms, G.R., Burnham, A.K., and Flygare, W.H., "Measurement of the dispersion in polarizability anisotropies," Journal of Chemical Physics, Vol. 63, 1975, pp. 3321-3326.

${ }^{26}$ Bridge, N.J., and Buckingham, A.D., “The polarization of laser light scattered by gases," Proceedings of the Royal Society A, Vol. 295, No. 1442, 1966, pp. 334-349.

27 Oddershede, J., Svendsen E.N., "Dynamic polarizabilities and Raman intensities of $\mathrm{CO}, \mathrm{N}_{2}, \mathrm{HCl}$, and $\mathrm{Cl}_{2}$," Chemical Physics, Vol. 64, No. 3, 1982, pp. 359-369. 